[16] V. I. Utkin and K. D. Young, "Methods for constructing discontinuous planes in multidimensional variable structure systems," Automat. Remote Contr., vol. 9, no. 10, pp. 1466-1470, 1978.

[17] P. H. Petkov, N. D. Christov, and M. M. Konstantinov, "A computational algorithm for pole assignment of linear multiinput systems," IEEE Trans. Automat. Contr., vol. AC-31, no. 11, pp. 1044-1047, 1986.

[18] M. Arnold and B. N. Datta, "An algorithm for the multinput eigenvalue assignment problem," IEEE Trans. Automat. Contr., vol. 35, no. 10, pp. $1149-1152,1990$.

\section{Transient Analysis of Nonlinear Circuits by Combining Asymptotic Waveform Evaluation with Volterra Series}

Mustafa Celik, Abdullah Atalar, and Mehmet A. Tan

Abstract-A new method is proposed for the transient analysis of circuits with large number of linear lumped elements and lossy coupled transmission lines, and with few mildly nonlinear terminations. The method combines the Volterra-series technique with Asymptotic Waveform Evaluation approach and corresponds to recursive analysis of a linear equivalent circuit.

\section{INTRODUCTION}

The Volterra functional series [1] have been widely used in the analysis of weakly nonlinear circuits because of their nice properties: they are noniterative and computationally efficient [2]. However, most of the previous research were concentrated on the frequency domain analysis [3]-[6], and to the best of our knowledge, no work has been reported so far which uses Volterra-series methods in the transient analysis.

The Asymptotic Waveform Evaluation (AWE) technique [7] ap proximates the dominant poles and residues of a linear circuit very efficiently using a moment matching technique. It has been generalized in such a way to handle distributed elements with frequency domain parameters [8] as well as to generate all of the dominant poles within the frequency range of interest [9], [10]. The AWE technique has been also extended for the nonlinear circuits [11], [12]. However, these approaches require a nonlinear search, usually by means of a Newton-Raphson iteration.

In this brief, we propose a fast method for the transient analysis of mildly nonlinear circuits containing elements specified with frequency domain parameters which cannot be analyzed using standard numerical integration algorithms. This method combines the Volterra series analysis of nonlinear circuits with the AWE technique. The proposed method corresponds to successive analysis of a linearized circuit with different excitations.

Manuscript received February 15, 1994; revised February 6, 1995. This work was supported by NATO-SFS project (TU-MIMIC). This paper was recommended by Associate Editor M. M. Green.

M. Celik was with the Department of Electrical and Electronics Engineering, Bilkent University, 06533 Bilkent, Ankara, Turkey. He is now with the Department of Electrical and Computer Engineering, University of Arizona, Tucson, AZ 85721 USA.

A. Atalar and M. A. Tan are with the Department of Electrical and Electronics Engineering, Bilkent University, 06533 Bilkent, Ankara, Turkey.

IEEE Log Number 9413215.

\section{THE VolterRa Series}

Consider a single-input single-output nonlinear system with a time domain relation,

$$
x(t)=f(w(t))
$$

where $x(t)$ is output, $w(t)$ is input and $f(\cdot)$ is a real-valued function. The output $x(t)$ can be expressed as a Volterra functional series of the input $w(t)$ in the form

$$
x(t)=\sum_{n=1}^{\infty} x_{n}(t)
$$

where the $n$th order output is related to the input as

$$
\begin{aligned}
x_{n}(t)= & \int_{-\infty}^{\infty} \cdots \int_{-\infty}^{\infty} h_{n}\left(\tau_{1}, \cdots, \tau_{n}\right) w\left(t-\tau_{1}\right) \cdots \\
& \cdot w\left(t-\tau_{n}\right) d \tau_{1} \cdots d \tau_{n} .
\end{aligned}
$$

It can be shown that the series (2) is convergent and the first few terms of the expansion is generally sufficient to represent the output if the system is mildly nonlinear. The multidimensional function $h_{n}\left(\tau_{1}, \cdots, \tau_{n}\right)$ in (3) is the $n$th order Volterra kernel or the nonlinear impulse response of the system of order $n$. The determination of the kernels - whether algebraically or numerically, is generally very difficult. Instead, the $n$th order output can be found directly using the relation,

$$
x_{n}(t)=\left.\frac{1}{n !}\left(\frac{d^{n}}{d \rho^{n}} f(\rho w(t))\right)\right|_{\rho=0} .
$$

In the next part of the paper, we propose a method to find the Volterra series of a general nonlinear circuit. For this purpose we will use the relation (4). As will be shown later, this relation, in fact, corresponds to repetitive analysis of a linearized circuit with different sources each time.

\section{THE METHOD}

\section{A. Circuit Formulation}

Consider a circuit $\mathcal{N}$ which contains linear lumped elements, linear subcircuits, and mildly nonlinear terminations. The subcircuits may contain elements specified with frequency domain parameters such as lossy coupled transmission lines. Suppose that all the linear subcircuits are grouped into a single subcircuit $\mathcal{N}_{\mathcal{A}}$. Let the subcircuit $\mathcal{N}_{\mathcal{A}}$ be represented by frequency domain terminal equations

$$
\mathbf{I}_{A}(s)=\mathbf{Y}_{A}(s) \mathbf{V}_{A}(s)
$$

where $\mathbf{I}_{A}$ and $\mathbf{V}_{A}$ are the $s$-domain terminal currents and voltages of $\mathcal{N}_{\mathcal{A}}$, respectively, and $\mathbf{Y}_{A}(s)$ is the admittance matrix in the $s$-domain.

Without loss of generality, the modified nodal analysis matrix [13] equation of the overall circuit $\mathcal{N}$ can be written as [14]

$$
\mathbf{C} \frac{d \mathbf{x}(t)}{d t}+\mathbf{G} \mathbf{x}(t)+\mathbf{D}_{A} \mathbf{i}_{A}(t)+\mathbf{f}(\mathbf{x}(t))=\mathbf{w}(t), \quad \text { for } t \geq 0
$$

where $\mathbf{x}(t)$ is a vector of size $N$ containing the waveforms of the node voltages, independent voltage source currents, linear inductor currents, nonlinear capacitor charges, and nonlinear inductor fluxes; $\mathbf{w}(t)$ is a vector containing the source waveforms; $\mathbf{C}$ and $\mathbf{G}$ are constant matrices formed by linear lumped components; $f(x)$ is a function describing the nonlinear elements; $\mathbf{D}_{A}$ is a selector matrix, 
whose entries are 1 or 0 , that maps $i_{A}(t)$ into the node space of the circuit. The relation of node voltages of the circuit to the node voltages of the subcircuit $\mathcal{N}_{\mathcal{A}}$ can be shown by the equation [14]

$$
\mathbf{v}_{A}(t)=\mathbf{D}_{A}^{T} \mathbf{x}(t),
$$

where the superscript $T$ denotes the transpose.

\section{B. Finding the Volterra Responses}

Now, let us replace the source vector $\mathbf{w}(t)$ with $\rho \mathbf{w}(t)$ and write the output vector, $\mathbf{x}(t)$, as a sum of Volterra functional series in the form

$$
\mathbf{x}(t)=\sum_{n=1}^{\infty} \mathbf{x}_{n}(t)
$$

where $\mathbf{x}_{n}(t)$ is the $n$th order output of the circuit,

$$
\mathbf{x}_{n}(t)=\left.\frac{1}{n !}\left(\frac{d^{n}}{d \rho^{n}} \mathbf{x}(t)\right)\right|_{\rho=0} .
$$

If we take the derivative of (6) with respect to $\rho$ and evaluate at $\rho=0$, we obtain a set of linear differential equation for the vector of the first order outputs,

$\mathbf{C} \frac{d \mathbf{x}_{1}(t)}{d t}+\mathbf{G} \mathbf{x}_{1}(t)+\mathbf{D}_{A} \mathbf{i}_{A 1}(t)+\mathbf{f}_{\mathbf{x}} \mathbf{x}_{1}(t)=\mathbf{w}_{1}(t)$, for $t \geq 0$ where $\mathbf{w}_{1}(t)=\mathbf{w}(t)$, and $\mathbf{f}_{\mathbf{x}}$ is a matrix of size $N$ by $N$ :

$$
\left[\mathbf{f}_{\mathbf{x}}\right]_{i j}=\left.\frac{\partial f_{i}}{\partial x_{j}}\right|_{\mathbf{x}=\mathbf{0}} .
$$

Taking the derivative of (6) with respect to $\rho$ once more gives $\mathbf{C} \frac{d \mathbf{x}_{2}(t)}{d t}+\mathbf{G} \mathbf{x}_{2}(t)+\mathbf{D}_{A} \mathbf{i}_{A 2}(t)+\mathbf{f}_{\mathbf{x}} \mathbf{x}_{2}(t)=\mathbf{w}_{2}(t), \quad$ for $t \geq 0$ where

$$
\mathbf{w}_{2}(t)=-\left(\frac{d \mathbf{f}_{\mathbf{x}}}{d \rho}\right) \cdot \mathbf{x}_{1}(t)
$$

and its $k$ th element is

$$
\left[\mathbf{w}_{2}(t)\right]_{k}=-\sum_{i=1}^{N} \sum_{j=1}^{N} \frac{\partial^{2} f_{k}}{\partial x_{i} \partial x_{j}}\left[\mathbf{x}_{1}(t)\right]_{i}\left[\mathbf{x}_{1}(t)\right]_{j} .
$$

Similarly, taking the derivatives of (6) with respect to $\rho$ repeatedly, it can be shown that the higher order outputs in (8) can be obtained by solving the same linear differential equation set with different source vectors each time,

$\mathbf{C} \frac{d \mathbf{x}_{n}(t)}{d t}+\mathbf{G} \mathbf{x}_{n}(t)+\mathbf{D}_{A} \mathbf{i}_{A n}(t)+\mathbf{f}_{\mathbf{x}} \mathbf{x}_{n}(t)=\mathbf{w}_{n}(t)$, for $t \geq 0$

and with the initial conditions, $\mathbf{x}_{n}(0)=0$. As a summary, the transient of a nonlinear circuit can be found very efficiently by computing the transient responses of a linear equivalent circuit repetitively for different sources.

\section{Finding the Source Vectors}

The $n$th order excitation vector, $\mathbf{w}_{n}(t)$, is a function of $\mathbf{x}_{i}(t)$ 's, $i=1, \ldots, n-1$, and derivatives of $\mathbf{f}(\mathbf{x})$ with respect to $\mathbf{x}$ evaluated at $\mathbf{x}=\mathbf{0}$ up to order $n$. The expression of $\mathbf{w}_{2}(t)$ is given in (14). However, it is cumbersome to represent the recursive dependence of higher order sources on the lower order outputs. The procedure for computing the excitation at the $n$th order circuit can be best explained at element level. This procedure is called the method of nonlinear currents and the details can be found in [3].

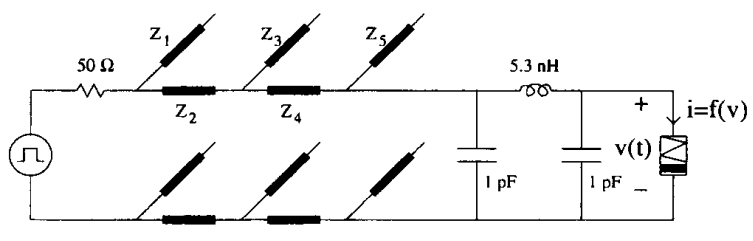

Fig. 1. Circuit for example \#1.

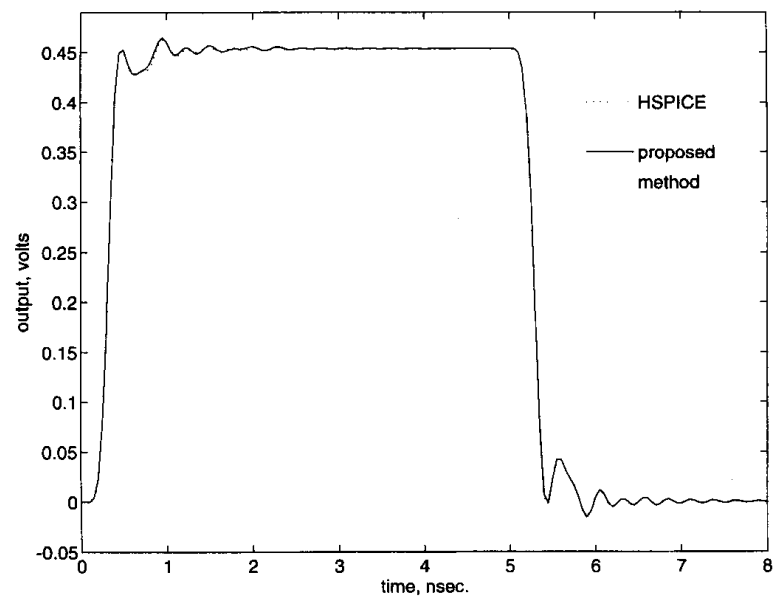

Fig. 2. The output voltage waveform for example \#1.

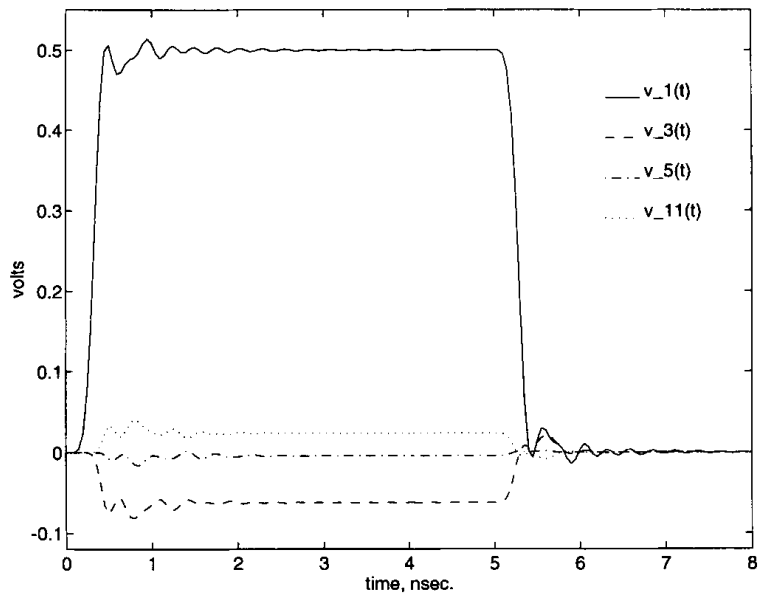

Fig. 3. First, third, fifth, and eleventh order outputs for example \#1.

\section{Finding the Transient Response of the Linearized Circuit}

To find the transient responses of the linearized circuit we use the asymptotic waveform evaluation technique. Using (5) and (7), the set of time domain equations given in (15) for the $n$th order circuit can be represented in Laplace domain as

$$
\left[s \mathbf{C}+\mathbf{G}+\mathbf{D}_{A} \mathbf{Y}_{A}(s) \mathbf{D}_{A}^{T}+\mathbf{f}_{\mathbf{x}}\right] \mathbf{X}_{n}(s)=\mathcal{L}\left\{\mathbf{w}_{n}(t)\right\}
$$

where we have assumed that $\mathbf{x}(0)=0$. From (16), we can write the output waveform as

$$
\mathbf{x}_{n}(t)=\int_{0}^{t} \mathbf{w}_{n}(t-\tau) \mathbf{z}(\tau) d \tau
$$




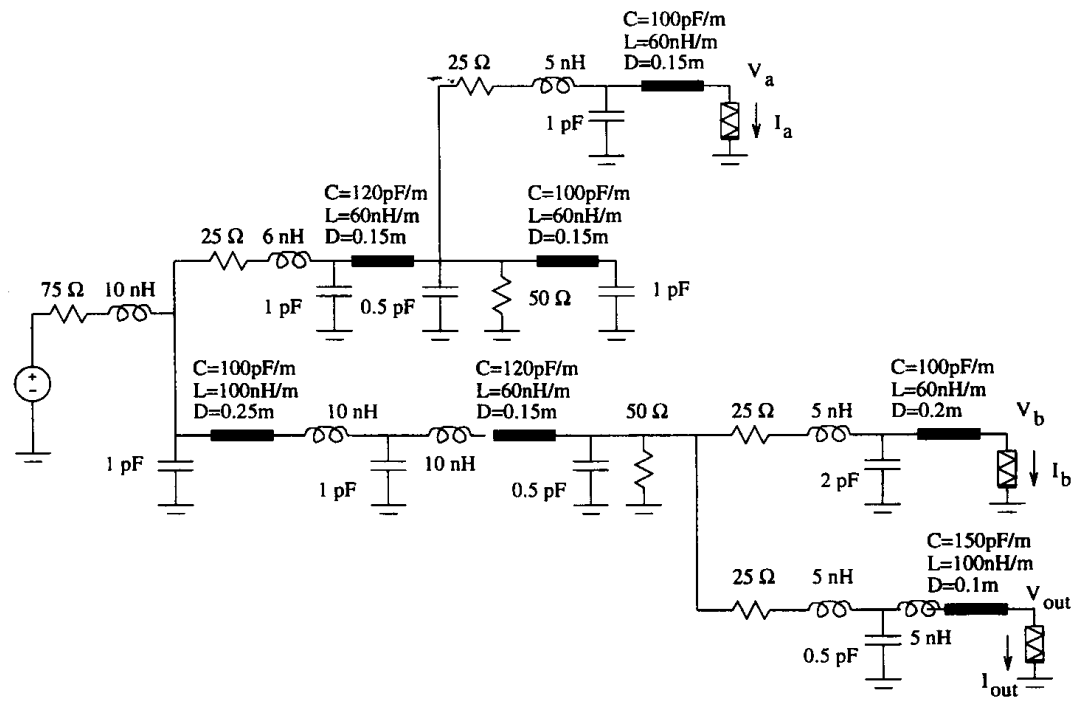

Fig. 4. Circuit for example \#2.

where the matrix valued impulse response

$$
\mathbf{z}(t)=\mathcal{L}^{-1}\{\mathbf{Z}(s)\}
$$

and

$$
\mathbf{Z}(s)=\left(s \mathbf{C}+\mathbf{G}+\mathbf{D}_{A} \mathbf{Y}_{A}(s) \mathbf{D}_{A}^{T}+\mathbf{f}_{\mathbf{v}}\right)^{-\mathbf{1}}
$$

We approximate $\mathbf{Z}(s)$ with a $q$-pole model using the asymptotic waveform evaluation technique,

$$
[\hat{\mathbf{Z}}(s)]_{i j}=\sum_{l=1}^{q} \frac{k_{i j l}}{s-p_{i j l}},
$$

or equivalently,

$$
[\hat{\mathbf{z}}(t)]_{i j}=\sum_{l=1}^{q} k_{i j l} e^{p_{i j l} t} .
$$

To increase the efficiency a single set of poles can be used for the whole circuit, rather than using a different set of poles for each entry. However, this approach would reduce accuracy as discussed in [15]. Moreover, it is not necessary to calculate the dominant poleresidue set for every matrix entry. Assuming that there is only one input and one output, we need $\left(N_{c}+1\right) N_{f}+N_{c}+1$ pole sets, where $N_{f}$ is the number of nonzero entries in $\mathbf{f}(\mathbf{x}(t))$ and $N_{c}$ is the number of elements in $\mathbf{x}(t)$ which are controlling variables of nonlinear elements, i.e., the arguments of $\mathbf{f}(\mathbf{x}(t))$. Therefore, the efficiency of the proposed method decreases with increasing $N_{f}$ and $N_{c}$. This implies that our method performs best for circuits with relatively few nonlinear elements where $N_{f}$ and $N_{c}$ are small.

In the proposed method we deal with the values of signals at some time points, not the exact waveforms. The signals are assumed to be linear functions of time between successive time points. Thus, any input waveform can be decomposed into a set of ramp functions. The ramp response of the circuit can be found symbolically using the approximated impulse response poles and residues. The output response then can be found in an efficient manner by shifting and scaling the ramp response [15].

We note that the source vectors in the proposed method are obtained in the time domain, by multiplying lower order output waveforms. The multiplications in the time domain spread the spectrum in

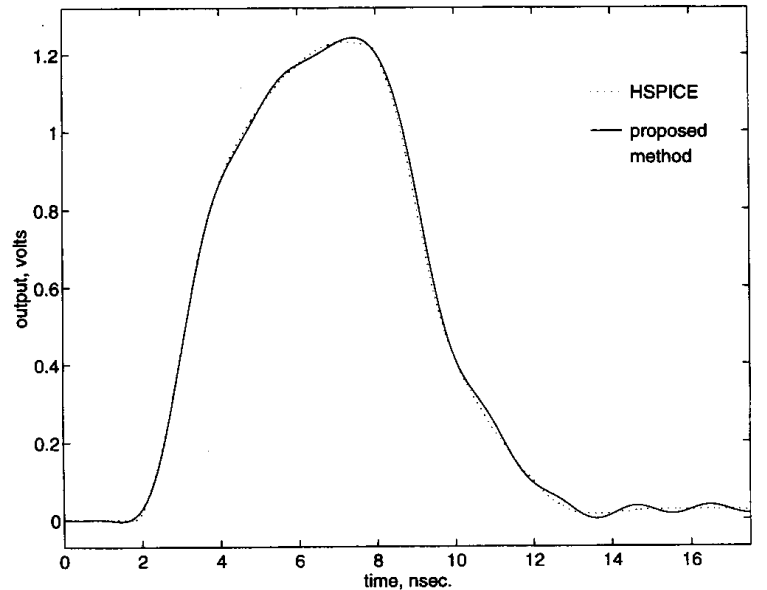

Fig. 5. The output voltage waveform for example \#2.

the frequency domain. Consequently, if a single point low-frequency AWE approximation is used to find the response of the linearized circuit, it produces error even in the low-frequency components of the source vectors and the error propagates in the recursive process. Therefore, multipoint moment matching techniques such as complex frequency hopping [9] or multipoint Padé approximation [10], must be applied to find the impulse response poles and residues. These techniques produce accurate results for very broad frequency regions and therefore the error is minimized.

\section{E. Examples}

Example 1: The first example is a low-pass filter implemented with transmission lines which is shown in Fig. 1. All transmission line sections are $\lambda / 8$ long at $f=4 \mathrm{GHz}$. The characteristic impedances are: $Z_{1}=Z_{5}=64.9 \Omega, Z_{2}=Z_{4}=217.5 \Omega, Z_{3}=70.3 \Omega$. The filter is terminated by a load which is defined by the nonlinear function, $i=v+v^{3}$. The input is a $5 \mathrm{~ns}$ pulse with $0.1 \mathrm{~ns}$ rise and fall times. In the first step of the analysis, the circuit is linearized by 
replacing the nonlinear load by a $1 \Omega$ resistor. The dominant impulse response poles and residues of the circuit are then calculated using AWE technique. For this circuit, two sets of poles and residues are required: from input to output and from a current source connected parallel to load to output. The first order response is then obtained using the first set of pole-residue by exciting the circuit with the actual input. Subsequently, the input is killed and the higher order responses are calculated by exciting the circuit with a current source connected parallel to load and using the second set of pole-residue. The order is increased until the algorithm converges. The output waveforms, obtained with the proposed method and HSPICE, are given in Fig. 2 for comparison. The first, third, fifth, and eleventh order outputs are shown in Fig. 3.

Example 2: The second example, which is shown in Fig. 4, has been taken from [16]. The nonlinear elements are defined as: $I_{a}=$ $0.001 V_{a}^{3}, I_{b}=V_{b} / 750+0.002 V_{b}^{3}$, and $I_{\text {out }}=0.001 V_{\text {out }}^{3}$ The applied input voltage waveform for this circuit is $4.5 \mathrm{~ns}$ pulse with $1.5 \mathrm{~ns}$ rise and fall times. The amplitude of the input pulse is 5 voits. The output waveform obtained using the proposed method is compared with HSPICE result in Fig. 5.

\section{CONCLUSION}

A new method has been proposed for the transient analysis of circuits with relatively few and mildly nonlinear terminations. In this approach, the method of Volterra-series analysis of the nonlinear elements is combined with AWE-based techniques for the linear part of the circuit. The method is noniterative and corresponds to recursive analysis of a linear circuit with different excitations. Therefore, it has no convergence problem. Since it is based on AWE technique, it uses a very small number of LU decompositions with respect to the traditional methods.

\section{REFERENCES}

[1] V. Volterra, Theory of Functionals and of Integral and IntegroDifferential Equations. New York: Dover, 1959.

[2] M. B. Steer, C. Chang, and G. W. Rhyne, "Computer-aided analysis of nonlinear microwave circuits using frequency-domain nonlinear analysis techniques: the state of the art," Int. J. Microwave Millimeter-Wave Computer-Aided Eng., vol. 1, pp. 181-200, 1991.

[3] S. L. Bussgang, L. Ehrman, and J. W. Graham, "Analysis of nonlinear systems with multiple inputs," Proc. IEEE, vol. 62, pp. 1088-1119, Aug. 1974.

[4] L. O. Chua and C.-Y. Ng, "Frequency-domain analysis of nonlinear systems: general theory," Electron. Circuits Syst., vol. 3, pp. 165-185 July 1979.

[5] _ _ "Frequency-domain analysis of nonlinear systems: formulation of transfer functions," Electronic Circuits Syst., vol. 3, pp. 257-269, Nov. 1979.

[6] S. Maas, Nonlinear Microwave Circuits. Norwood, MA: Artech House, 1988

[7] L. T. Pillage and R. A. Rohrer, "Asymptotic waveform evaluation for timing analysis," IEEE Trans. Computer-Aided Design, vol. 9, pp. 352-366, Apr. 1990

[8] T. K. Tang and M. S. Nakhla, "Analysis of high-speed VLSI interconnects using the asymptotic waveform evaluation technique," IEEE Trans. Computer-Aided Design, vol. 11, pp. 341-352, Mar. 1992

[9] E. Chiprout and M. Nakhla, "Transient waveform estimation of highspeed MCM networks using complex frequency hopping," in Proc. Multi-Chip Module Conf., Mar. 1993.

[10] M. Celik, O. Ocali, M. A. Tan, and A. Atalar, "Pole-zero computation in microwave circuits using multipoint Padé approximation," IEEE Trans. Circuits Syst., vol. 42, pp. 6-13, Jan. 1995.
[11] V. Raghavan and R.A. Rohrer, "AWESpice: A general tool for the efficient and accurate simulation of interconnect problems," in Proc. Design Automation Conf., June 1992.

[12] E. Chiprout and M. Nakhla, Asymptotic Waveform Evaluation and Moment Matching for Interconnect Analysis. Boston: Kluwer, 1993.

[13] C. W. Ho, A. E. Ruehli, and P. A. Brennan, "The modified nodal approach to network analysis," IEEE Trans. Circuit Theory, vol. CT-22, pp. 504-509, June 1975.

[14] R. Griffith and M. S. Nakhla, "Mixed frequency/time domain analysis of nonlinear circuits," IEEE Trans. Computer-Aided Design, vol. 11 pp. 1032-1043, Aug. 1992.

[15] T. K. Tang, M. S. Nakhla, and J. R. Griffith, "Analysis of lossy multiconductor transmission lines using the asymptotic waveform evaluation technique," IEEE Trans. Microwave Theory Tech., vol. 39, pp. 2107-2116, Dec. 1991.

[16] S. Lum, M. S. Nakhla, and Q-J. Zhang, "Sensitivity analysis of lossy coupled transmission lines with nonlinear terminations," IEEE Trans. Microwave Theory Tech., vol. 42, pp. 607-615, Apr. 1994.

\section{On Lyapunov Control of the Duffing Equation}

\author{
Henk Nijmeijer and Harry Berghuis
}

Abstract-In this brief, we develop feedback control strategies for a chaotic dynamic system such as the Duffing equation. Our controllers are of the so-called Lyapunov-type and are inspired by robot manipulator feedback controls. The different controllers we propose include observerbased controllers that even can cope with parametric uncertainties of the original system. Some simulation examples support the developed methods.

\section{INTRODUCTION}

Recently, an increasing interest has been developed in controling chaotic nonlinear systems as arising in physics and engineering; from the various relevant references we mention [4]-[8], [12], [13], [15], and references therein. A very essential element in the control of chaos is that, in many cases, the ultimate goal of control is to decrease random effects and to stabilize the system at an equilibrium point, or more general, about a given reference trajectory. In such cases, one is in fact naturally led to reduce or even more completely annihilate the chaotic dynamics that an uncontrolled system may exhibit. Depending on the specific desired behavior of the system, several methods for controlling chaotic systems have been proposed, see, e.g., [6], [13]. Among the methods given there, a prominent role is played by the socalled Lyapunov-type methods. At the same time and earlier, various authors have investigated stabilizing control schemes for second-order mechanical systems, as in particular robot manipulators. Let us give a sample of relevant references [1], [10], [14], [17], [19], noting that also this field is strongly progressing at the moment. It should be noted that also in this context Lyapunov-type methods are very popular and useful.

The purpose of this paper is essentially to develop a controllerobserver scheme for controlling a chaotic second-order system such as

Manuscript received September 8, 1994; revised February 27, 1995. This paper was recommended by Associate Editor G. Chen.

H. Nijmeijer is with the University of Twente, Department of Applied Mathematics, 7500 AE Enschede, The Netherlands.

H. Berghuis is with Hollandse Signaalapparaten B.V., Department RDTR\&S-MFE-SER, 7550 GD Hengelo, The Netherlands.

IEEE Log Number 9413209. 